\title{
WIRELESS SENSOR NETWORKS: APPLICATIONS UTILIZING SATELLITE LINKS
}

\author{
Ioannis Mathioudakis \\ University of Southampton \\ Southampton, United Kingdom
}

\author{
Neil M. White \\ University of Southampton \\ Southampton, United Kingdom
}

\author{
Nick R. Harris \\ University of Southampton \\ Southampton, United Kingdom
}

\section{ABSTRACT}

Wireless sensor networks offer unique opportunities for applications in the human and natural environments. In this paper, the types of application, which utilize satellites, are explored. Also, a proportional differentiation model is proposed in order to provide the quality of service over satellite networks. We implement and evaluate a new dropper, called Satellite Proportional Dropper, to successfully apply this model on satellite links.

\section{INTRODUCTION}

Advancements in wireless communications and MicroElectro-Mechanical Systems (MEMSs) have enabled the development of low-cost, low-power, multifunctional, tiny sensor nodes that can sense the environment, perform data processing and communicate to each other over shortdistances. This progress, together with marked advances in the area of microsensors has allowed small, inexpensive, energy-efficient and reliable sensors with wireless networking capabilities to become a reality. The development of such devices has given rise to the increasingly popular concept of wireless sensor networks (WSNs), which has been the subject of extensive studies and has enabled a wide range of applications. [1]

In this paper, we discuss application areas that need satellite links. Such areas include environmental and habitat monitoring, satellite remote sensing for ocean research and structural health monitoring. The objectives of this work are to explore the aforementioned applications and, since satellite bandwidth is expensive and limited, there is a need for a framework, which can make optimum use of these. We therefore propose the Proportional Differentiation Model (PDM, based on a concept described by Dovrolis [2], [3]) on satellite links in order to provide relative differentiated services. In addition, this model is investigated in terms of performance metrics, which in our case is the proportional loss rate. Finally, we carry out simulations, using the software tool Network Simulator 2 (NS-2) [9], to verify that the PDM architecture can be successfully applied to satellite links.

In Section 2, the problem statement is defined and we describe sensor applications that utilize the satellite links. The Satellite Proportional Dropper (SPD) algorithm is introduced in Section 3 in order to justify the model and the evaluation results of SPD algorithm are presented in Section 4.

\section{APPLICATIONS AREAS}

\section{A. Problem Statement}

Satellite bandwidth is very expensive, so networks need to be able to support different degrees of quality of service for different applications. Moreover, with the rapid acceleration of network traffic and with applications varying from realtime to non real-time, service providers offer different levels of service, based on business priorities of the users or applications. We therefore propose the proportional differentiation model (PDM) in order to provide the service differentiation, since different users have different needs. The PDM architecture, which was first proposed by Dovrolis [2], is successfully achieved in terrestrial and wireless architectures and our key goal is to apply it over satellite links. This model is a lightweight architecture providing relative quality of service to a finite number of service classes. This architecture states that certain class performance metrics should be proportional to the differentiation parameters that the provider chooses. A generic description of this model follows. Suppose that $\bar{q}(t, t+\tau)$ is a performance measure of class $i$ and $j$ in the interval $(t, t+\tau)$, where $\tau>0$ is the monitoring timescale. As we are interested in differentiation over short timescales, the value of $\tau$ should be relatively small. So, the PDM form for all pairs of classes and for all the time intervals is defined:

$$
\frac{\bar{q}_{i}(t, t+\tau)}{\bar{q}_{j}(t, t+\tau)}=\frac{c_{i}}{c_{j}}
$$

where $c$ is the generic quality differentiation parameter. Thus, in our case the proportional loss rate differentiation takes the form:

$$
\frac{\bar{l}_{i}(t, t+\tau)}{\overline{l_{j}}(t, t+\tau)}=\frac{\sigma_{i}}{\sigma_{j}}
$$

where $\sigma$ is the loss rate differentiation parameter. The properties of PDM are controllable, since the network operators can adjust the quality spacing between different traffic classes, depending on unique pricing and policing criteria and predictable in the sense that the class differentiation should be consistent, such as higher classes should receive better performance than lower, even if for a short time and also even if there are changes in class loads. In addition, the benefits of this model include the ability to maintain scalability in the network, adjust and eliminate the performance gap, which means that one class will receive most resources of the network and the other classes will share the remaining resources. Finally, the grade of differentiation between classes can be controlled according to quality differentiation parameters, which are pre-specified by the network operators. However, these parameters must be associated with differentiation loss rate, which in this work is 
the performance metric of each class. [3]

Thus, we consider a satellite network with $\mathrm{N}$ satellite gateway terminals, serving $\mathrm{M}$ different classes of services, each one associated with a loss rate differentiation parameter, $\mathrm{L}_{\mathrm{i}}$. Each gateway terminal is connected with a wireless ad-hoc sensor network in order to transmit the sensing data to other regions. These terminals are the sources and the receivers in the network respectively. Each time a packet arrives, a loss scheduling scheme is applied to decide whether to discard the arriving packet or add it in the buffer. Hence, the loss scheme is a control policy that determines if a packet is to be dropped and which packet to drop, whenever a new packet arrives. Moreover, a loss scheme can be space conserving, if a packet is discarded only when the buffer is full and space nonconserving, if a packet may be discarded even when the buffer is not full. An example of space conserving is the Drop-Tail algorithm. Examples of space non-conserving algorithms include the RED and SPD respectively. [5] Let us propose that $L_{i}(t ; C ; \pi)$ is the number of lost packets for the class $i$ by time $\mathrm{t}$ given that the channel capacity is $\mathrm{C}$ and the loss scheme adopted is $\pi$ and stationary, because the control policy does not change over the time. We suppose that all the arrival processes are stationary $\left\{A_{i}(t), t \geq 0\right\}$, so it is assumed that the loss rate $f_{i}(t, C, \pi)$ is calculated by the form: [4]

$$
f_{i}(t, C, \pi)=L_{i}(t, C, \pi) / A_{i}(t)
$$

The proportional differentiation model is applied for first time in satellite networks. All the previous studies are in the field of wired and wireless network. Thus, before introducing the SPD algorithm, it is necessary to state clearly the differences between our case and existing research:

- Most other studies have assumed the existence of a co-located buffer, while in satellite networks the terminals (nodes) are distributed and do not have the status knowledge of each other.

- To maintain a network wide differentiation with distributed terminals is much more complex than those used for terrestrial networks, whereby the focus is simply on a single router sharing buffer.

- The different arrival rate of each source.

- The different buffer length of each satellite terminal.

\section{B. Sensor Applications Utilizing Satellites}

A sensor network is composed of a large number of small nodes that have sensing, processing and wireless communications capabilities. The advantage of using these networks is that system installation and maintenance cost is remarkably reduced and also no cables are required for data transfer, as the communication is wireless and the data processing can be distributed across the network nodes. Moreover, the system becomes more tolerant, since in the case of a partial system failure the rest of the system is capable of performing its task independently. The concept of integration of sensor networks and satellite networks is to transmit various types of sensing data in different places, where cable and terrestrial infrastructure cannot economically be installed and maintained. The main advantages of using satellite networks are global coverage, bandwidth allocation, broadcast and multicast capability and rapid deployment. The main application areas utilizing satellites are environmental and habitat monitoring, satellite remote sensing for ocean research and structural health monitoring.

Habitat and environmental monitoring represent a class of sensor network applications with enormous potential benefits for scientific communities and society as a whole. Deploying natural spaces with numerous networked micro-sensors can enable long-term data collection at scales and resolutions that are difficult, if not impossible, to obtain otherwise. The main components of a typical habitat-monitoring application are: the samples originate at the sensor nodes, which typically involve heterogeneous sensing capability, processing power, and storage. They are typically deployed in dense patches, where each patch corresponds to a particular slice of the habitat of interest. The data from the various patches flow through the transit network to an on-site data centre. Satellite gateway terminals play a key role in transmission of sensing data from places like rural and urban region, rivers, deserts, isolated islands, ships etc., to monitoring centre. [7]

The increased pollution on the ocean has been causing concern and a major threat to sustaining the biological richness of oceans and coastal areas. Therefore, there is a need for long-term management and sustainable development of ocean resources. The advent of satellites which provide repetitive and wide-area coverage has radically changed the nature of oceanographic observations in recent years for the retrieval of many ocean geophysical parameters and understanding of their interlinkages more scientifically. Utilizing the advantages offered by satellite remote sensing, significant progress has been achieved in the retrieval of various oceanographic processes. The main sensors that are being used for satellite oceanographic observations are passive, (i.e. the sensor receives energy naturally reflected by or emitted from the earth's surface), or active, (i.e. the sensor provides its own illumination) and records the amount of incident energy returned from the imaged surface. This application provides a wealth of information on a diverse range of geophysical and biological parameters and phenomena. [6]

Built environments of civil infrastructures, such as bridges, tunnels or buildings, experience gradual deterioration over their life span due to factors such as corrosion, fatigue or earthquakes. So, periodic monitoring can be used to provide information about to the structural health of the infrastructure over its operational life and give an alarm in the case of special climate, special load or serious abnormal operation. In such a system, multiple kinds of massive monitoring data describing the state of structures must be acquired and processed simultaneously and in timely way. The process of continuously monitoring, the status of structure to detect damage is defined as structural health monitoring [8]. 


\section{SAtellite Proportional Dropper Algorithm}

It is necessary to take into account several assumptions in order to apply the PDM architecture and to cope with the problems we stated in section 2, before we describe the SPD algorithm. Thus, we suppose that the satellite network needs to support $\mathrm{M}$ services classes and each class is associated with a loss differentiation parameter $\sigma$, which in our dropper is the Quality of Service (QoS) Label. These labels are provided by the network operators, so the problems of distributed nodes, different buffer lengths of each terminal and the different arrival rates of each source are successfully solved, since every class is now serving according to a pre-defined performance. Moreover, an SPD dropper is attached to each gateway and the satellite domain is assumed to be well dimensioned, which means that at normal operation, the load in the network is well routed or balanced, so the average buffer length of each satellite terminal is maintained at a certain level. In the case that the network utilization is low the SPD dropper does not apply, as it is meaningless to drop packets and to provide the service differentiation.

In figure 1 is illustrated the functionality with a numerical example of SPD algorithm. Each class is defined by three parameters, which are the QoS-label, the minimum threshold $\left(\mathrm{Thr}_{\min }\right)$ and the maximum threshold $\left(\mathrm{Thr}_{\max }\right)$.

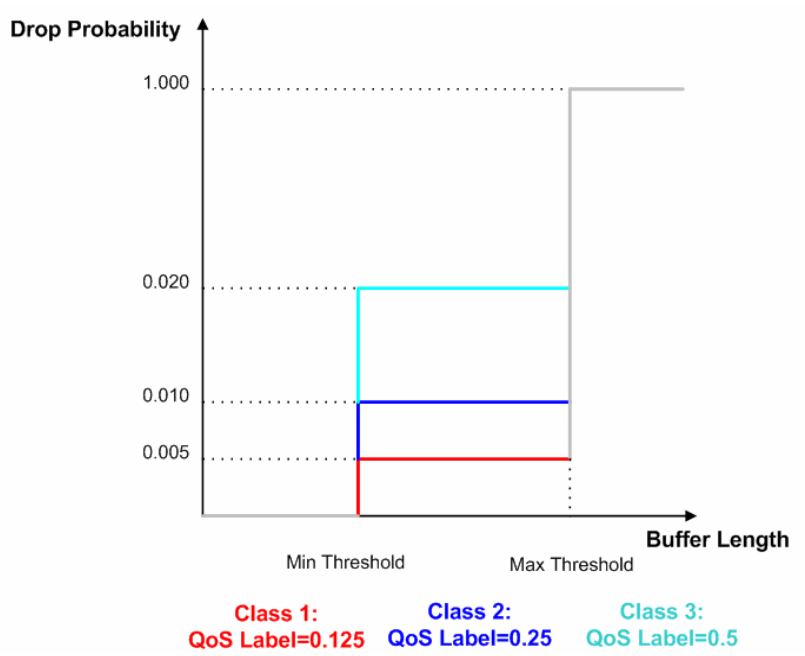

Figure 1: A numerical example, which shows the functions of SPD. The QoS labels of these classes are 0.125, 0.25 and 0.5 respectively and the reference drop probability is set at 0.04 . Hence, the drop probability for each class, which is calculated by equation (4), is $0.005,0.01$ and 0.02 respectively.

The QoS-label defines the priority of every class and takes values from 0 to 1 . The value 0 is the ideal, where we suppose that the packets never drop and 1 is the worst priority, where all the packets are dropped according to a reference drop probability, which is configured by the network operator. Moreover, SPD utilizes two queue thresholds, the minimum threshold $\left(\mathrm{Thr}_{\min }\right)$ and the maximum threshold $\left(\mathrm{Thr}_{\max }\right)$ to determine the congestion level. Thus, if the average queue size is below the lower threshold the drop probability for each class is null, so the network is at the normal operation, and when it is above the higher threshold the drop probability is 1 , therefore all the arriving packets are dropped. Once the average queue length is between the two thresholds, the arriving packet may be served according to the QoS-label parameter. This parameter defines the dropping scheme for every traffic class, so the drop probability (drop_prob) of each class comes from the following form:

$$
\text { drop }_{-} \text {prob }_{i}=Q o S-\text { label }_{i} \times p
$$

where $p$ is the reference drop probability. We set $p$ at 0.04 , which means that the algorithm drops one out of 25 of the arriving packets, because SPD performs better when the drop probability changes fairly slowly as the average queue size changes and this also helps to eliminate oscillations in the average queue size. There should never be a reason to set $p$ greater than 0.1 (one out of 10 of the arriving packets), since then the average queue size will be close to the maximum threshold, so the size of queue cannot be controlled. Additionally, it is necessary to configure the minimum and the maximum thresholds. The optimal values for these depend on the desired average queue size and as discussed earlier the network utilization must be very high in order to provide the differentiation. Thus, the minimum threshold must be correspondingly large to allow network utilization to be maintained at a high level. Hence, we set the minimum threshold at $40 \%$ and the maximum threshold at $80 \%$ of the buffer length respectively. [5]

The QoS labels are pre-specified by the network operator, and different QoS-label for each class means different dropping probabilities and therefore different values of packet loss and loss rates, respectively. If the QoS-labels of one pair of classes are proportional, then we expect the loss rates of the same pair to be proportional as well, and to satisfy the form:

$$
l_{i} / l_{j}=Q o S-\text { label }_{i} / Q o S-\text { label }_{j}
$$

\section{Evaluation of Results}

The SPD algorithm is attached to each satellite gateway terminal in order to provide the relative differentiated service and to achieve the PDM architecture. Each terminal is linked with ad-hoc sensor networks in order to transmit the sensing data to other places. These terminals are connected with other terminals through a geostationary (GEO) satellite. In this scenario eight satellite terminals are installed, with four sources and four receivers respectively. We evaluate our model under an exponential traffic generation, because we want to count only the absolute number of dropped packets. The capacity of each satellite link is 2,048 Mbps and the buffer length is 1000 packets and the transmission rate of each terminal is 2.1 Mbps. Typically, the capacity of the satellite link is $1.5 \mathrm{Mbps}$ to transmit the sensing data to other places. The minimum and the maximum thresholds are configured at 400 and 800 packets respectively. The reference drop probability is set at 0.04 . In our case, four different 
traffic classes are provided by the network operator, with QoS labels $0.125,0.25,0.5$ and 1.0 respectively. We chose these labels, since the ratio between the two pairs of classes (e.g. Class $1 /$ Class 2, Class 2/Class 3 etc) is 0.5 and it is expected then the ratio of loss rate between the same pairs will tend to 0.5 . Finally, all the experiments are done through the NS-2.

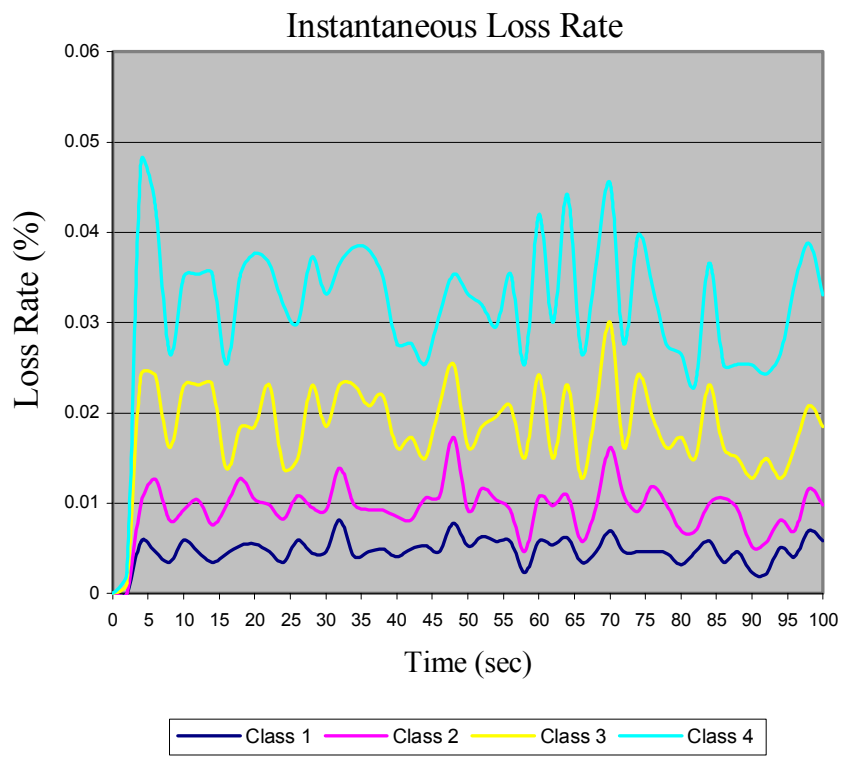

Figure 2: In this graph is illustrated the instantaneous loss rate for each class. The loss rate is estimated by dividing packets that were dropped from those that arrived in a monitoring period.

Figure 2 illustrates the instantaneous loss rate for each class. The SPD dropper is attached to each satellite terminal in order to apply the proportional differentiation model. The loss rate is calculated by dividing packets that were dropped by those that arrived, during the same interval $(t, t+\tau)$. So, the first observation is that the first class has the best priority and the fourth class has the worst. The loss rate of each class is approximately double the loss rate of the next lower class, thus the relative differentiated service is provided over satellite links. The performance gap between the classes is controlled according to QoS labels, so each class receives the pre-defined performance by the network operator. Finally, with the SPD dropper the PDM architecture is successfully applied after short period (in 5 seconds), since the buffer of each terminal is empty at the beginning of simulation, so the dropper cannot operate.

Figure 3 is important as it shows the instantaneous loss rate ratio for the pairs of classes (which are Class 1/Class 2 and Class 3/Class 4). The points on each chart show their shortterm loss rate, and the values are between 0.4 and 0.6 , so they are very close to the ideal ratio, which is set up from QoS labels in 0.5 . For the same case, the loss rates in this figure are not equal to the ideal ratio, and there are occasional deviations. But, this is logical, because it is related to the feasibility of PDM architecture. If the packet loss in an interval of arrivals is too small (in this case 2 seconds), it may not be possible to drop enough packets from each class according to the QoS labels, so that the loss rates are not proportionally adjusted. In either case the deviations of each graph will decrease, as the monitoring interval increases.

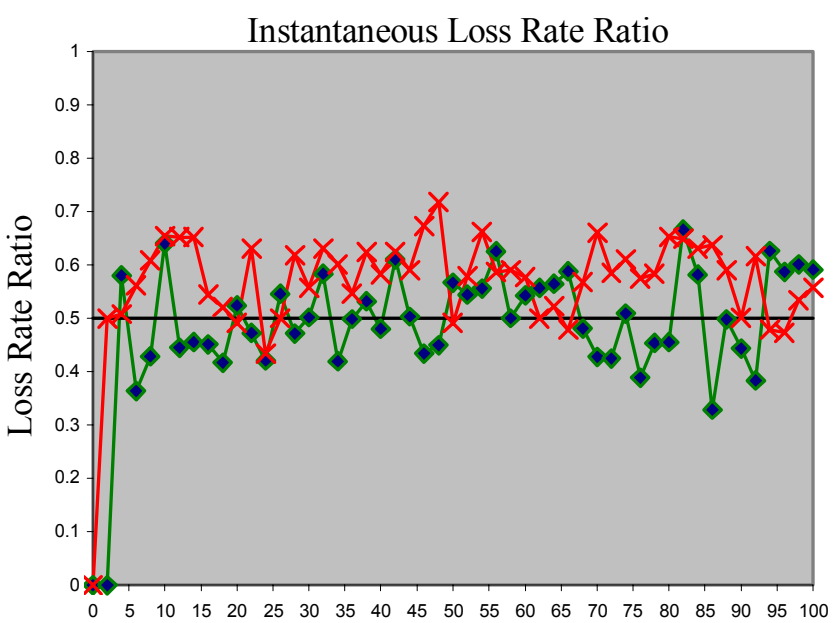

Time (sec)

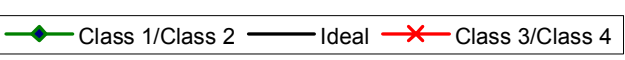

Figure 3: Instantaneous loss rate ratio for Class $1 / 2$ and Class $3 / 4$

However, let us change the previous scenario in order to better validate the SPD algorithm. It is therefore assumed that the pairs of classes $\{1,2\}$ and $\{3,4\}$ have the same QoS labels respectively. We set the labels for the two pairs of classes at 0.125 and 0.5 respectively. All the other parameters from the first scenario remain the same. In figure 4, the results of this scenario are illustrated. The instantaneous loss rate for the pairs $\{1,2\}$ and $\{3,4\}$ is approximately similar, as expected. Of course, there are infrequent deviations, but the resources of the network are shared relatively.

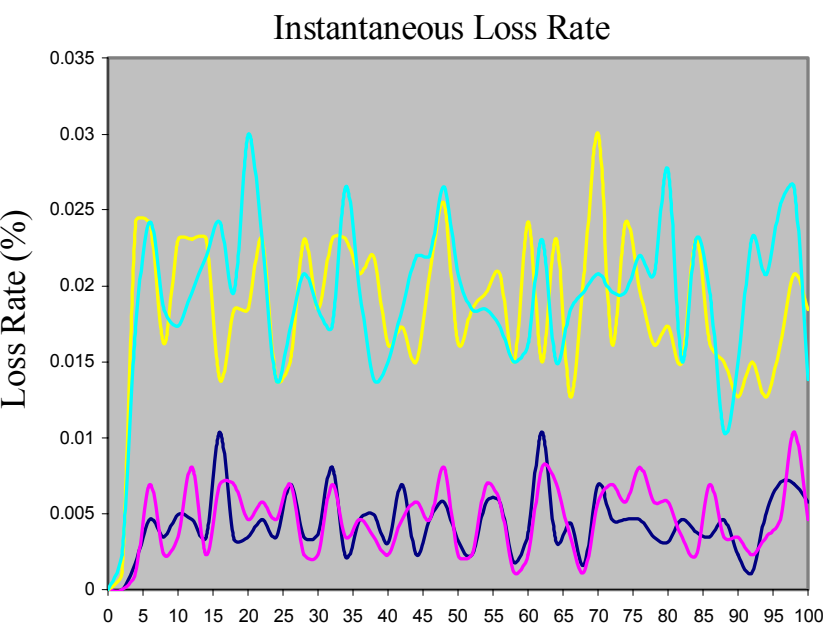

Time (sec)

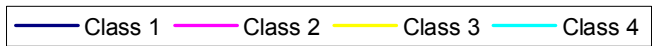

Figure 4: Instantaneous loss rate is almost similar for the classes 1, 2 and 3, 4

In addition, table 1 depicts the total packet loss of each traffic class for the two scenarios. In the first scenario the 
ratio for class 1 and 2 is $48.44 \%$ and for 3 and 4 is 54\% respectively, which is very close to our target, while in the second scenario the ratio for the same pairs of classes is $108 \%$ and $94.9 \%$ respectively.

Table 1: Total packet loss

\begin{tabular}{|l|c|c|}
\hline \multicolumn{1}{|c|}{ Classes } & $\begin{array}{c}\text { Scenario 1 } \\
\text { (packets) }\end{array}$ & $\begin{array}{c}\text { Scenario 2 } \\
\text { (packets) }\end{array}$ \\
\hline Class 1 & 202 & 217 \\
\hline Class 2 & 417 & 201 \\
\hline Class 3 & 809 & 802 \\
\hline Class 4 & 1498 & 845 \\
\hline
\end{tabular}

Finally, figure 5 shows a comparison of loss rates between SPD, Drop-Tail and RED algorithm. Each dropper is applied to each satellite terminal. The setting parameters for this scenario remain the same, as we described at the beginning of this section.

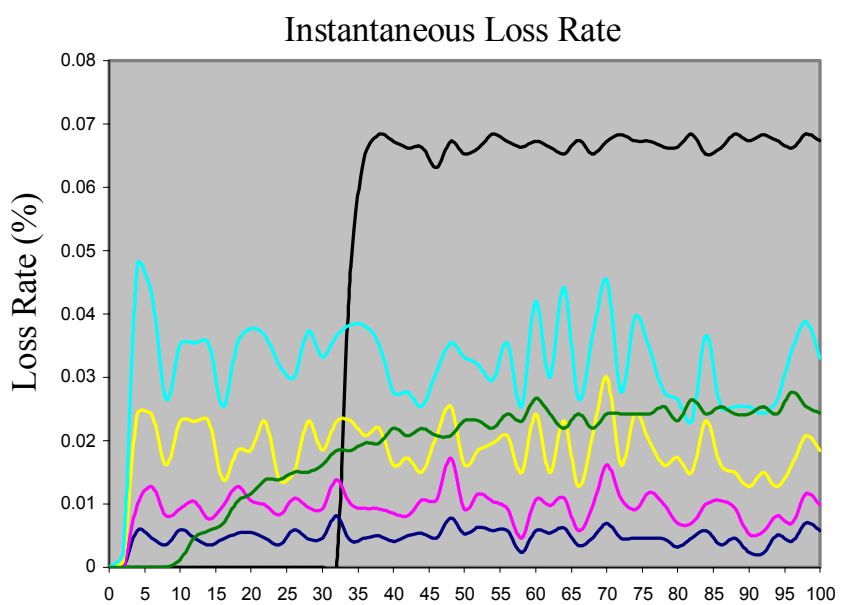

Time $(\mathrm{sec})$

\begin{tabular}{ll}
\hline Drop-Tail & Class $1-$ Class 2 \\
Class 4 & RED
\end{tabular}

Figure 5: Comparison of loss rates between the Satellite Proportional Dropper, Drop-Tail and RED droppers (default).

The function of Drop-Tail is that it first stores the internal packet, and then checks the buffer length. Once the buffer overflows, the most recently packet is dropped. As can be seen the loss rate is zero in the first 32 seconds, as in this period the buffer does not overflow. After that period, it is almost constant at 0.07 until the simulation ends. The packets are dropped randomly. However, the function of RED is such that when the average queue size is less than then minimum threshold, no packets are marked and if it is greater than the maximum threshold all the arriving packets are marked. When the average queue size is between the two thresholds each arriving packets are marked (dropped) with probability $p_{a}$, where $p_{a}$ is the function of the average queue size [10]. The performance of RED is much better than the Drop-tail and the fourth class of SPD, but is worse than Class 1, 2 and 3. The main difference between these droppers is that the
SPD can provide different traffic classes with different priorities, while the other droppers can provide only one. Also, one crucial point is the configuration of priority for the transmitting information. Since some types of sensing data may be very important for us, so we do not want to lose it. One solution to this problem is to propose that all the important data will be sent with best priority. Of course, we can not guarantee zero loss rates for the high priority class, but we can guarantee that the performance for the higher class will be better than the other classes.

\section{CONClusions}

In this paper, the key goal was to study the effect of applying PDM architecture over satellite links. It was a very challenging topic, since all the earlier studies were limited to the field of terrestrial and wireless networks. The main contribution of this paper is the implementation and evaluation a new type of dropper, called the Satellite Proportional Dropper (SPD) in order to provide service differentiation over satellite links. Moreover, we compared SPD with the Drop-Tail and RED droppers using the software tool NS-2. The novelty with this dropper is the ability to define the performance of each class according to the QoS labels. With this dropping scheme the problems we stated in section 2 are solved. Furthermore, the PDM is successfully applied in satellite links, because the SPD algorithm meets the main criteria of this architecture, which are controllability and predictability. Finally, network operators can provide these services for their customers, based on their demands and pricing criteria. One further suggestion for this work is to investigate the PDM architecture in terms of different performance metrics, such as throughput.

\section{REFERENCES}

[1] F. Zhao and L. Guibas, Wireless Sensor Networks: An information processing approach. San Francisco: Morgan Kaufmann Publishers, 2004, pp. 1-21.

[2] C. Dovrolis and P. Ramanathan, "A case for relative differentiated services and the proportional differentiation model", IEEE Network, vol. $13,5,1999$, pp. 26-34.

[3] C. Dovrolis and P. Ramanathan, "Proportional differentiated services, part II: loss rate differentiation and packet dropping", Eighth International Workshop on Quality of Service, IWQOS, 2000, pp. 5361.

[4] T. Yang and J. Pan, "A measurement-based loss scheduling scheme", Fifteen Annual Joint Conference of the IEEE Computer Societies, Networking the Next Generation, Proceedings IEEE INFOCOM, vol. 3, 1996, pp. 1062-1071.

[5] S. Floyd and V. Jacobson, "Random Early detection gateways for congestion avoidance", IEEE/ACM Transactions on Networking, vol. 1, 4, 1993, pp. 397-413.

[6] L. C. Smith, "Satellite remote sensing of river inundation area, stage and discharge: a review", Hydrological processes, vol. 11, 10, 1997, pp. 1427-1439.

[7] R. Szewczyk, E. Osterweil, J. Polastre, M. Hamilton, A. Mainwaring and D. Estrin, "Habitat monitoring with sensor networks", Communications of the ACM, vol. 47, 6, 2004, pp. 34-40.

[8] Z. F. Gao, Y. L. Du, M. B. Su and B. Chen, "Network sensors and its application in structure health monitoring system", First International Conference on Innovative Computing, Information and Control, vol. 1, 2006, pp. 68-71.

[9] NS-2.29 Simulator, available from: http://www.isi..edu/nsnam/ns . 